\title{
Estudo morfológico dos fibroadenomas da mama: uma análise comparativa entre grupos etários
}

\section{Morphologic study of the breast fibroadenomas: a comparative analysis between age groups}

Sandra Simon Calado'; Maria do Carmo Carvalho de Abreu e Lima²

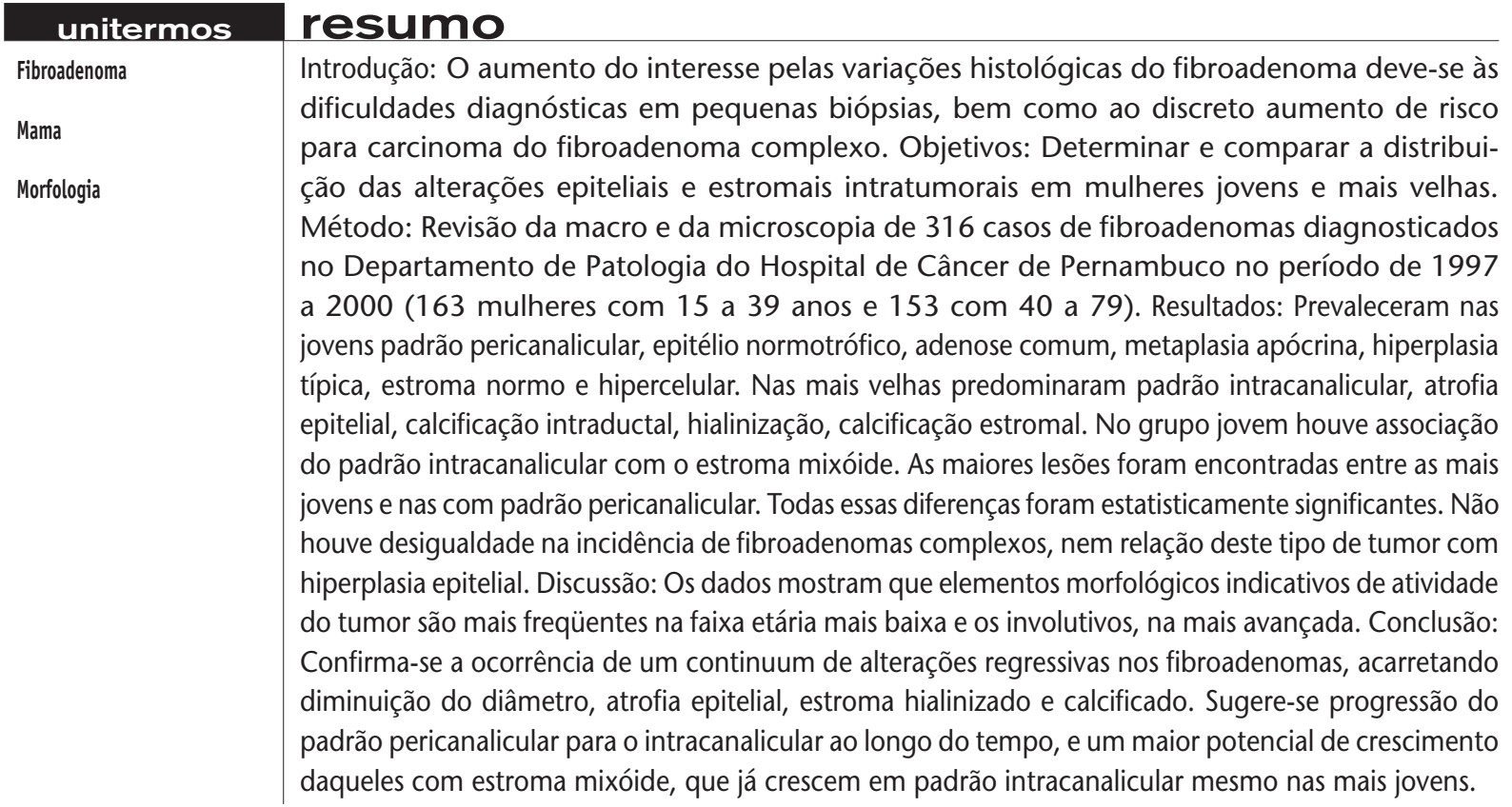

Background: The increased interest in histological variations of fibroadenomas is due to the diagnostic difficulties found in small biopsies, as well as the slightly increased risk for carcinoma in cases of complex fibroadenoma. Goal: Determine and compare the distribution of intratumoral epithelial and stromal alterations in young and older women. Method: Revision of gross and microscopy of 316 cases of fibroadenomas diagnosed in Department of Pathology at Hospital de Cancer de Pernambuco, from 1997 to 2000 (163 women with 15 to 39 years and 153 with 40 to 79 years). Results: Pericanalicular pattern, normotrophic epithelium, adenosis, apocrine hyperplasia, typical hyperplasia, normo and hypercelular stroma prevailed in the younger group. Intracanalicular pattern, epithelial atrophy, intraductal calcification, stromal hyalinization and calcification were predominant in the older group. There was an association between intracanalicular pattern and myxoid stroma. The larger lesions were more commonly found in younger group as well as in cases showing pericanalicular pattern. These findings were statistically significant. There was no difference in incidence of complex fibroadenomas. Discussion: Morphological elements that indicate lesion activity were more common in younger women, whereas involutive findings were more frequent in older group. Conclusion: The occurrence of a continuum of regressive alterations in fibroadenomas is confirmed by decrease in diameter, epithelial atrophy, stromal hyalinization and calcification. The study also suggests there is a progression from pericanalicular to intracanalicular pattern along the years, as well as a larger growth potential for those showing myxoid stroma, which is associated with intracanalicular pattern even in younger women.

1. Mestra em Anatomia Patológica pela Universidade Federal de Pernambuco (UFPE); chefe do Laboratório da Anatomopatologia do Hospital da Aeronáutica do Recife. 2. Professora-adjunta; doutora do Departamento de Patologia do Centro de Ciências da Saúde (CCS) da UFPE.

Trabalho realizado no Departamento de Patologia do CCS/UFPE, Programa de Pós-Graduação em Anatomia Patológica, e baseado na tese de mestrado intitulada Estudo Morfológico dos Fibroadenomas da Mama, com Ênfase à Análise Comparativa entre as Faixas Etárias de 15 a 39 Anos e de 40 a 79 Anos, apresentada em maio de 2003 no CCS/UFPE. 


\section{Introdução}

Nos últimos anos tem-se observado um interesse renovado ${ }^{(14,21)}$ no estudo dos fibroadenomas. Em grande parte, essa tendência deveu-se aos resultados dos trabalhos desenvolvidos por Dupont et al.(9), que demonstraram a associação desses tumores com um risco relativo de 3,1 para o câncer de mama quando presentes determinadas alterações intralesionais. Os fibroadenomas mostram um espectro muito rico e variado de alterações epiteliais e estromais que podem sofrer modificações ao longo do tempo, refletindo o millieu hormonal a que estão expostos. No nosso meio, apesar de constituir lesão freqüente na prática clínica e na rotina do anatomopatologista, o fibroadenoma tem sido pouco estudado, particularmente no que tange à variedade de alterações consideradas de risco para o câncer.

Classificado dentro das lesões mamárias bifásicas, este tumor se manifesta a partir da proliferação de elementos epiteliais e estromais, podendo ser considerado lóbulo de tamanho excessivo, formado a partir de um crescimento descoordenado e exagerado dos dois elementos desencadeado por estímulos hormonais desequilibrados num tecido suscetível à proliferação ${ }^{(4,12,22)}$. Clinicamente se apresenta como um pequeno nódulo indolor que cresce lentamente até um a três centímetros, mas que pode atingir grandes proporções ${ }^{(15)}$.

O método diagnóstico primário dos fibroadenomas é a mamografia, entretanto, apesar de oferecer sensibilidade de $85 \%$ a $95 \%{ }^{(23)}$, a especificidade deste exame é pequena, sendo normalmente necessários procedimentos diagnósticos adicionais. A realização de biópsia estereotática por agulha contribui muito para o diagnóstico diferencial das lesões mamárias, com altas taxas de sensibilidade e especificidade, mesmo em lesões não-palpáveis ${ }^{(1,2)}$, permitindo o acompanhamento preferencial dessas lesões ao invés de sua excisão cirúrgica.

O tumor é constituído por subunidades epiteliais redondas ou ovais semelhantes a lóbulos, cada uma delas formada por duas camadas de células epiteliais e mioepiteliais circundadas por estroma. O crescimento do estroma em torno de ductos tubulares ou a sua compressão sobre eles resulta nos padrões peri e intracanalicular, respectivamente ${ }^{(17,18)}$.

O elemento estromal do fibroadenoma pode sofrer várias modificações, como hialinização, calcificação, aumento focal ou difuso da celularidade e, ainda, alterações mixóides, que o transformam num nódulo gelatinoso. Nas mulheres mais velhas, os fibroadenomas mostram menor grau de celularidade, predominando a hialinização estromal, com freqüente calcificação. Já os tumores que apresentam celularidade estromal elevada são mais comuns em adolescentes da raça negra, demonstrando maior percentual de multiplicidade das lesões, com elevado risco de recorrência ${ }^{(10,18)}$.

A expressão morfológica do componente epitelial pode variar bastante, desde o seu completo desaparecimento até a presença de hiperplasia epitelial típica e atípica e, por fim, carcinoma in situ ou invasivo. A classificação dos fibroadenomas complexos entre as condições mamárias que elevam o risco de carcinoma mamário, como postulado por Dupont et al. ${ }^{(9)}$, é baseada em achados histológicos específicos, como calcificação intra-epitelial, cistos com mais de três milímetros, alteração papilar apócrina e adenose esclerosante. O epitélio dos ductos pode mostrar vários graus de hiperplasia, com ou sem atipismo. De regra, as hiperplasias, quando confinadas ao fibroadenoma, não implicam aumento do risco para câncer invasor ${ }^{(7,14,21)}$. O desenvolvimento de câncer dentro do fibroadenoma é raro, mas referido na literatura ${ }^{(18)}$, sendo mais da metade confinada ao fibroadenoma.

\section{Objetivos}

Descrever os padrões histológicos e as alterações epiteliais e estromais encontradas nos fibroadenomas, analisando possíveis diferenças entre esses componentes nas faixas etárias de 15 a 39 anos (grupo 1) e de 40 a 79 anos (grupo 2), com determinação do diâmetro tumoral médio em cada grupo. Os achados histológicos no corpus mammae circunvizinho ao tumor, quando em quantidade suficiente para análise, também serão descritos.

\section{Material e método}

O material para a presente pesquisa provém do arquivo do Departamento de Patologia do Hospital de Câncer de Pernambuco, de onde foram selecionados 163 blocos e lâminas das peças cirúrgicas de mulheres entre 15 e 39 anos (grupo 1) e de 153 entre 40 e 79 anos (grupo 2) com diagnóstico de fibroadenoma, perfazendo um total de 316 casos no período de 1997 a 2000.

Para a coleta de dados utilizou-se uma ficha padronizada contendo as diversas características histológicas do tumor. No momento da revisão das preparações histológicas não havia conhecimento da idade da paciente para que se evitasse avaliação tendenciosa. Em um segundo tempo, verificou-se a idade da paciente, obtendo-se também os dados macroscópicos de cada lesão. 
Para análise dos diferentes parâmetros foram utilizados critérios histológicos estabelecidos na literatura $(3,5,8,11,18,19)$. Estabeleceram-se critérios para o diagnóstico de:

1. área com aspecto phyllodes: setor de configuração foliácea por protrusão do estroma em ductos dilatados ou cistos. A celularidade estromal nestas áreas não poderia diferir da do restante do fibroadenoma ${ }^{(17,18)}$ (Figura 1A);

2. trofismo do componente epitelial: com base na aparência e na espessura do revestimento epitelial nas unidades ductolobulares dos fibroadenomas. Quando presente em todos os ductos, com espessura preservada, foi considerado normotrófico. Rotulou-se a lesão como normotrófica/atrófica quando o epitélio ora se mostrava bem visível, ora aplainado. A designação de epitélio atrófico ficou para aquelas lesões que exibiam epitélio achatado, atenuado. E quando os ductos não exibiam nenhum revestimento epitelial, o fibroadenoma teve seu epitélio dito desaparecido. Foram escolhidos casospadrão que serviram como referência para a avaliação dos demais;

3. fibroadenoma pericanalicular, intracanalicular ou misto: existência de $70 \%$ ou mais de área ocupada pelo mesmo padrão para definição dos primeiros. Quando o predomínio era menor ou quando não o havia, os tumores foram enquadrados como mistos (peri e intracanaliculares) (Figuras 1A e 1B);

4. alterações papilares apócrinas: projeções papilares de epitélio com características apócrinas para o lúmen do ducto (Figura 2A);

5. adenose comum: adensamentos excessivos de pequenas glândulas em arranjo lobulocêntrico, com dilatação freqüente daquelas mais periféricas, sem atipismos celulares ou desarranjo significativo da arquitetura ductolobular. O diagnóstico diferencial destes casos foi feito com área adenoma-símile em fibroadenoma, na qual uma região significativa do tumor estaria ocupada pela proliferação glandular tubular, tendendo a formar nódulos isolados, únicos, sem a característica lobulocêntrica da adenose $\mathrm{e}^{(17,18) \text {; }}$

6. adenose esclerosante: elevado número de elementos glandulares deformados e escleróticos, num arranjo lobulocêntrico, cobrindo uma área de pelo menos duas vezes o tamanho de uma unidade ductolobular normal|(13) (Figura 2B);

7. hiperplasia epitelial sem atipia: com o objetivo de evitar o risco de confundir tangencialidade do corte ou proliferação de células mioepiteliais com hiperplasia epitelial em ductos, levando a superestimação dos casos positivos para hiperplasia dentro do fibroadenoma, exigiu-se, neste estudo, a proliferação do epitélio com formação de tufos, pontes, arcadas ou fenestrações, sendo necessárias no mínimo quatro camadas de células epiteliais para se classificar o epitélio como hiperplásico (critério esse utilizado para o diagnóstico de hiperplasia moderada ou florida por Page $\left.{ }^{(16)}\right)$. A ausência de atipias nucleares, com cromatina fina e bem distribuída, e a orientação arquitetural variada classificaram a hiperplasia como típica. A extensão do processo foi categorizada como difusa quando houve hiperplasia em todos os quadrantes do tumor;

8. cistos: as cavidades foram mensuradas na própria lâmina, com régua transparente sobre a preparação histológica, especificando-se aquelas com tamanho superior a três milímetros (Figura $2 \mathrm{C}$ ). No intuito de diferenciar os cistos das fendas amplas, mais comuns e alongadas, apenas as cavidades de conformação arredondada ou ovóide foram consideradas;

9. microcalcificações: no componente epitelial foram separadas em intraductais e intra-epiteliais. As primeiras não têm qualquer continuidade com o epitélio de revestimento ductal, e as intra-epiteliais são aquelas que se depositam sobre as células epiteliais (Figura 2D). No estroma foram consideradas as calcificações de qualquer tamanho;

10. fibroadenomas complexos: seguindo-se os critérios de Dupont et al.(9), quando estavam presentes uma ou mais das seguintes características: adenose esclerosante, cistos com mais de três milímetros, simples ou apócrinos, calcificação epitelial e hiperplasia apócrina micropapilar;

11. estroma hialinizado: consideraram-se difusamente hialinizados aqueles casos com área maior ou igual a $70 \%$, e focalmente hialinizados os com valores inferiores a $40 \%$;

12. estroma mixóide: casos em que havia hipocelularidade e basofilia da matriz, vasos capilares lobulares proeminentes, com elementos celulares representados por escassas células fusiformes ou estreladas, de núcleo pálido. Tais características poderiam ocupar toda a extensão do tumor ou apenas os espaços intralobulares ${ }^{(6)}$;

13. estroma fibrocelular: os casos que não apresentavam estroma hialinizado ou mixóide foram subdivididos, quanto à celularidade, em hipo, normo e hipercelular. 


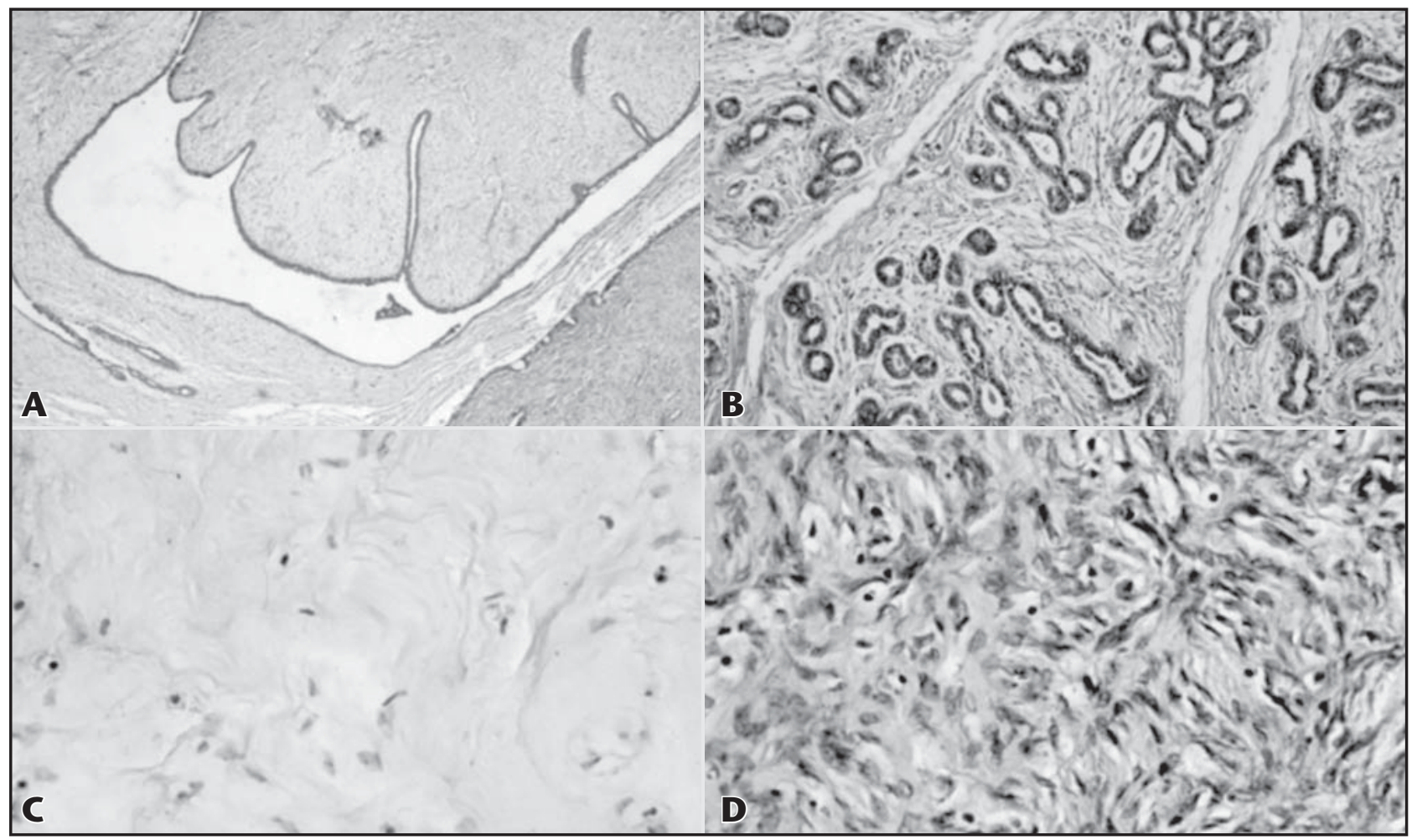

Figura 1 - A: Área com aspecto phyllodes em fibroadenoma. 0 aspecto intracanalicular exagerado e foliáceo da lesão, típico do tumor phyllodes, contrasta com a baixa celularidade estromal (HE 25x); B: fibroadenoma pericanalicular com ductos tubulares (HE 100x); C e D: casos usados como padrão para determinação de hipocelularidade (C), com raras células fusiformes imersas em matriz fibrosa, e hipercelularidade (D) estromal dos fibroadenomas, sem atipias ou figuras de mitose (HE 400x)

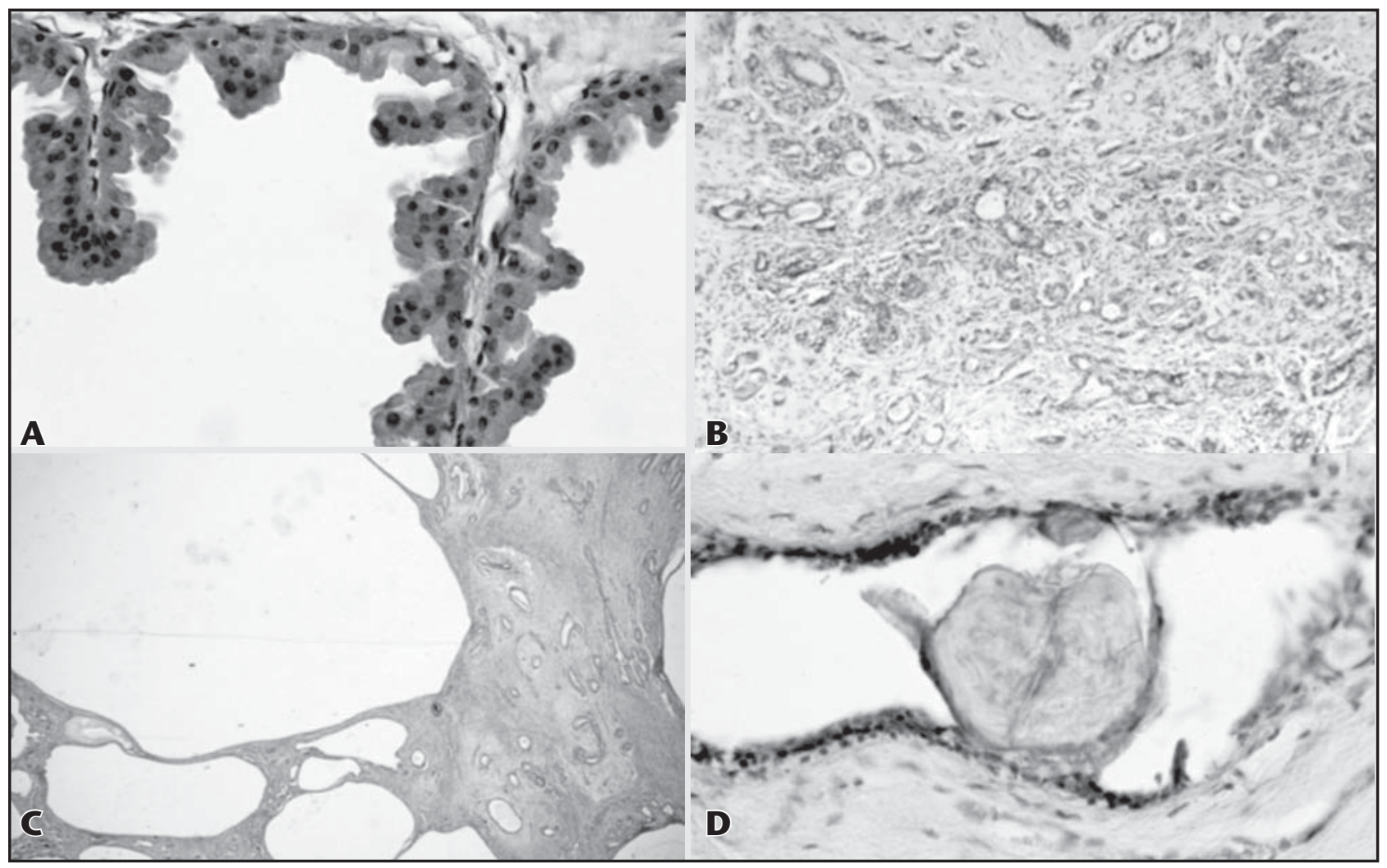

Figura 2 - A: Alteração apócrina papilar em fibroadenoma mostrando o delicado eixo fibrovascular circundado por células apócrinas (HE 400x); B: adenose esclerosante em fibroadenoma. Apesar do desarranjo arquitetural, há preservação da arquitetura lobulocêntrica (HE 100x); C: cistos intranodulares com mais de 3mm de diâmetro (HE 25x); D: calcificação intra-epitelial em fibroadenoma característica do fibroadenoma complexo (HE 100x) 
Foram selecionadas preparações histológicas que serviram como padrão de referência para a leitura das demais preparações estudadas (Figuras 1C e 1D). A hipercelularidade estromal foi definida como difusa quando correspondia a uma área maior ou igual a 50\% do fibroadenoma;

14. metaplasia adiposa: presença de adipócitos maduros em meio ao estroma usual do fibroadenoma, na região central do tumor. A infiltração adiposa foi determinada quando o tecido adiposo encontrava-se na periferia do fibroadenoma, penetrando-o de fora para dentro;

15. presença de rima tecidual circundando o fibroadenoma: se este tecido tivesse $0,5 \mathrm{~cm}$ de espessura ou se contivesse pelo menos cinco unidades lobulares ${ }^{(9)}$. As alterações de caráter proliferativo (hiperplasia típica moderada ou florida, hiperplasia atípica, adenose esclerosante e papilomatose) foram anotadas quando presentes.

Os dados são apresentados sob a forma de valores médios e distribuição de frequêencias conforme o tipo de variável. A análise estatística constou da utilização do teste qui-quadrado com a correção de Yates para as comparações de freqüências. Quando os dados não alcançavam os critérios necessários para o uso do qui-quadrado, utilizou-se o teste exato de Fisher. Para as comparações de médias foi adotado o teste $t$ de Student. Considerou-se um erro alfa de $5 \%(p<0,05)$ para rejeição da hipótese de nulidade.

\section{Resultados}

No grupo 1, a média de idade foi de $24,6 \pm 6,9$ anos, enquanto no grupo 2 foi de $53,7 \pm 8,1$ anos. $O$ diâmetro tumoral médio foi de $2,4 \pm 1,2 \mathrm{~cm}$ para o grupo 1 , enquanto no grupo 2 caiu para $1,8 \pm 0,9 \mathrm{~cm}(p<0,01)$. Os fibroadenomas pericanaliculares também foram significantemente maiores que os intracanaliculares $(2,3 \pm 1,37 \mathrm{~cm}$ versus 1,9 $\pm 1,02 \mathrm{~cm})$. Houve diferença estatística entre a média do diâmetro dos fibroadenomas mixóides do grupo das muIheres mais jovens e a dos não-mixóides no grupo geral, sendo maior no primeiro.

Entre os 316 fibroadenomas examinados, 61\% foram enquadrados como intracanaliculares, $28 \%$ como pericanaliculares e $11 \%$, mistos. A Tabela 1 mostra a distribuição dos padrões de crescimento entre os grupos. Observando-se o grupo 1, não houve predominância de fibroadenomas pericanaliculares em relação aos intracanaliculares.

$\mathrm{Na}$ Figura 3 explicita-se, entre os fibroadenomas intracanaliculares, o predomínio do estroma do tipo mixóide no grupo 1 e do tipo hialinizado no grupo $2(p<0,001)$.
A Figura 4 representa a distribuição dos tipos de epitélio descobertos nos fibroadenomas entre os grupos, e a distribuição dos diversos achados epiteliais encontrados nos fibroadenomas está relacionada na Tabela 2.

Encontrou-se área com aspecto phyllodes em dez tumores, sendo $80 \%$ deles nas pacientes com menos de 40 anos. Três dos fibroadenomas albergavam setor adenoma-símile na sua estrutura (todos no grupo 1). A presença de cápsula foi constatada em $17 \%$ dos casos, e $46 \%$ das lesões exibiam parênquima mamário circundante avaliável.

Dezenove por cento dos tumores foram classificados como complexos (59 casos), nove deles exibindo simultaneamente dois dos critérios de inclusão. Não houve diferença estatística na incidência de fibroadenomas complexos entre os dois grupos, bem como não existiu correlação entre complexidade dos fibroadenomas e presença de hiperplasia epitelial típica intratumoral.

Um caso de metaplasia escamosa do epitélio ductal em um cisto e um de papilomatose intraductal intratumoral foram encontrados, ambos no grupo 2. Hiperplasia secretória de tipo lactante foi um achado raro, encontrando-se apenas dois casos, ambos no grupo 1. Não houve diagnóstico de hiperplasia epitelial atípica intraductal ou intralobular, bem como foram ausentes os casos de carcinoma dentro de fibroadenoma nesta série.

O estroma fibrocelular predominou no grupo 1, com 99 casos (60\%), sendo 59 (36\%) normocelulares, $21(13 \%)$ hipocelulares e $19(11 \%)$ hipercelulares. Hialinização estromal foi verificada em 18 casos (11\%), e alterações mixóides, em 46 (28\%). Entre os fibroadenomas do grupo de mais idade 29 (19\%) tinham estroma fibrocelular (7\% normocelulares, $9 \%$ hipocelulares e $3 \%$ hipercelulares). Havia hialinização em 95 lesões (62\%), e as alterações mixóides foram menos freqüentes do que no grupo 1, com 19\% de incidência (29

\section{Distribuição dos padrōes de} crescimento dos fibroadenomas

Tabela 1 entre os grupos etários

\begin{tabular}{lccc} 
Padrão de & Grupo 1 & Grupo 2 & Total \\
crescimento & $n(\%)$ & $n(\%)$ & $n(\%)$ \\
Pericanalicular & $70(43)$ & $20(13)$ & $90(28)$ \\
Intracanalicular & $74(45)$ & $118(77)$ & $192(61)$ \\
Misto & $19(12)$ & $15(10)$ & $34(11)$ \\
Total & $163(100)$ & $153(100)$ & $316(100)$ \\
\hline
\end{tabular}

Houve diferença entre os padrões de crescimento nos dois grupos $(\mathrm{p}<0,001)$. 


\section{Tabela 2 Distribuição das alterações epiteliais dentro dos fibroadenomas nos grupos etários 1 e 2}

\begin{tabular}{|c|c|c|c|c|}
\hline Tipo de alteração & & $\begin{array}{c}\text { Grupo } 1 \\
n(\%)\end{array}$ & $\begin{array}{c}\text { Grupo } 2 \\
n(\%)\end{array}$ & $p^{*}$ \\
\hline \multirow{2}{*}{ Área adenoma-símile } & Sim & $3(2)$ & 0 & \multirow{2}{*}{$>0,05$} \\
\hline & Não & $160(98)$ & 153 & \\
\hline \multirow{2}{*}{ Adenose comum } & Sim & $10(6)$ & $1(0,6)$ & \multirow[t]{2}{*}{$<0,05$} \\
\hline & Não & $153(94)$ & $152(99,4)$ & \\
\hline \multirow{2}{*}{ Microcistos } & Sim & $36(22)$ & $21(14)$ & \multirow{2}{*}{$>0,05$} \\
\hline & Não & $127(78)$ & $132(86)$ & \\
\hline \multirow{2}{*}{ Metaplasia apócrina } & Sim & $46(28)$ & $20(13)$ & \multirow{2}{*}{$<0,0$} \\
\hline & Não & $117(72)$ & $133(87)$ & \\
\hline \multirow{2}{*}{ Calcificação intraductal } & Sim & $6(4)$ & $21(14)$ & \multirow[t]{2}{*}{$<0,01$} \\
\hline & Não & $157(96)$ & $132(86)$ & \\
\hline \multirow{2}{*}{ Hiperplasia epitelial típica focal } & Sim & $53(33)$ & $30(19)$ & \multirow{2}{*}{$<0,05$} \\
\hline & Não & $110(67)$ & $123(81)$ & \\
\hline \multirow{2}{*}{ Hiperplasia epitelial típica difusa } & Sim & $4(2)$ & $4(3)$ & \multirow{2}{*}{$>0,05$} \\
\hline & Não & $159(98)$ & $149(97)$ & \\
\hline
\end{tabular}

${ }^{*}$ Comparação entre os grupos 1 e 2 .

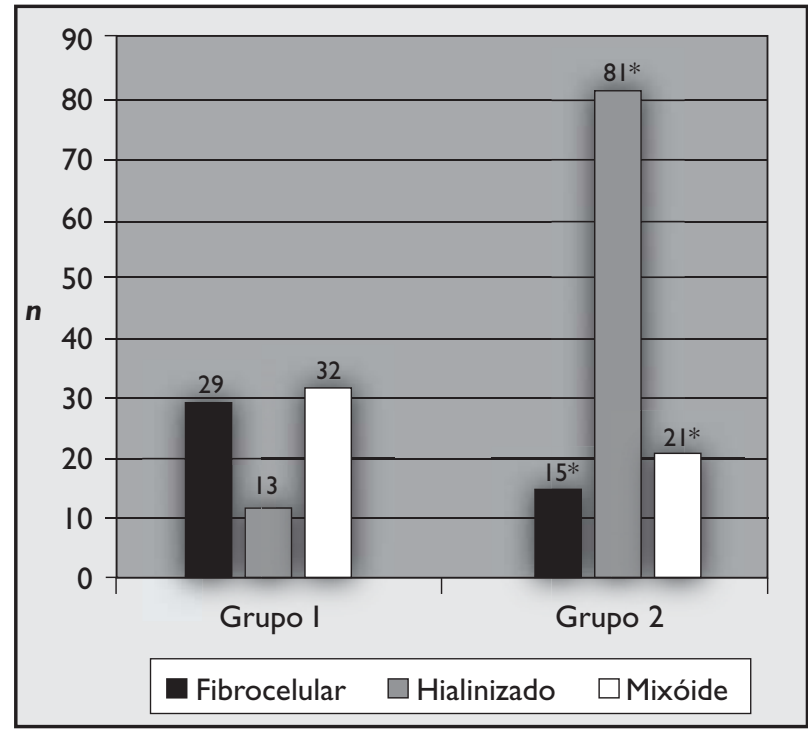

Figura 3 - Distribuição dos tipos de estroma entre os fibroadenomas

intracanaliculares nos dois grupos. Comparação entre os grupos 1 e 2. * $\mathrm{p}<0,001$

casos). Outro achado foi quanto à distribuição dos fibroadenomas com estroma mixóide entre os padrões peri e intracanalicular (Tabela 3), especialmente quando estudado o grupo 1 isoladamente (Tabela 4). Nele, o aspecto mixóide associou-se ao padrão intracanalicular em 32 casos (43\%), em contraste com os nove casos relacionados ao padrão pericanalicular $(12 \%)(p<0,001)$.

Três casos de infiltração adiposa foram encontrados no grupo 2, e seis de metaplasia adiposa se distribuíram

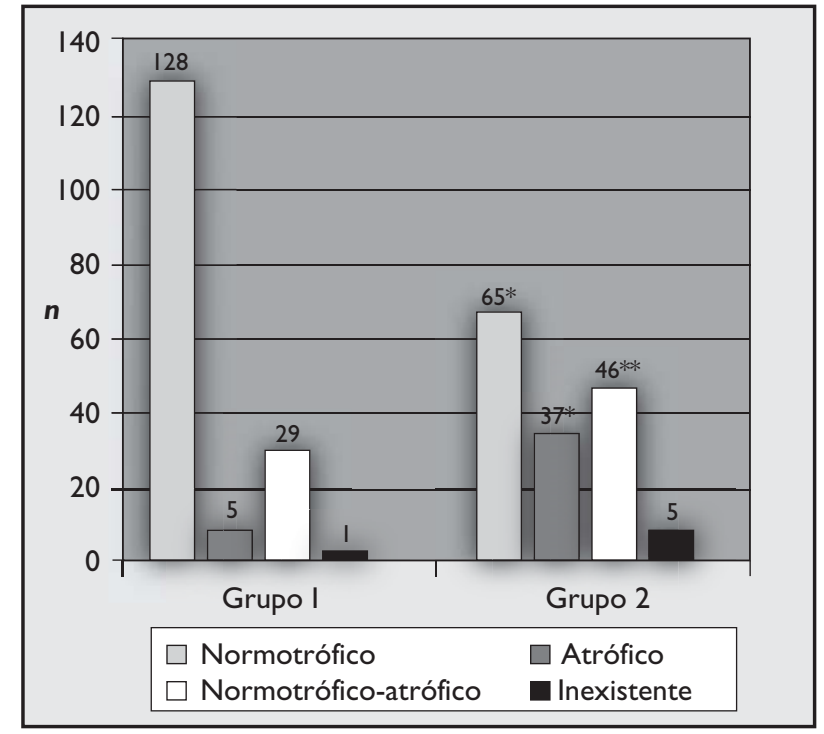

Figura 4 - Distribuição dos tipos de epitélio dos fibroadenomas nos dois grupos etários. Comparação entre os grupos 1 e 2. * $p<0,001$; ${ }^{* *} p<0,05$

nos dois grupos. Foi observada a presença de ossificação estromal em duas lesões, uma em cada grupo.

A diferença no número de casos de calcificação estromal entre os dois grupos foi bastante significante, com 32 casos $(21 \%)$ no grupo 2 e apenas um caso $(0,6 \%)$ no grupo 1 $(p<0,001)$.

O parênquima mamário adjacente ao fibroadenoma foi considerado avaliável em 147 lesões, 36,8\% dos casos do grupo 1 e $56,8 \%$ do grupo 2 . Não foi verificada relação 
Correlação entre presença $e$ ausência de estroma mixóide nos FAPC e nos FAIC na totalidade dos casos. Foram excluídos

Tabela 3 os fibroadenomas mistos

\begin{tabular}{lccc} 
Estroma & FAIC & FAPC & Total \\
mixóide & $n(\%)$ & $n(\%)$ & $n(\%)$ \\
Presença & $53(28)$ & $13(14)$ & $66(23)$ \\
Ausência & $139(72)$ & $77(86)$ & $216(77)$ \\
Total & $192(100)$ & $90(100)$ & $282(100)$ \\
\hline
\end{tabular}

Comparação entre FAIC e FAPC: $p<0,05$.

Correlação entre presença e ausência de estroma mixóide

Tabela 4 nos FAPC e nos FAIC no grupo 1

\begin{tabular}{lccc}
\hline Estroma & FAIC & FAPC & Total \\
mixóide & $n(\%)$ & $n(\%)$ & $n(\%)$ \\
Presença & $32(43)$ & $9(12)$ & $41(28)$ \\
Ausência & $42(57)$ & $61(8)$ & $103(72)$ \\
Total & $74(100)$ & $70(100)$ & $144(100)$ \\
\hline
\end{tabular}

Comparação entre FAIC e FAPC: $p<0,05$.

significativa entre a presença de alterações proliferativas (hiperplasia epitelial típica e atípica, papilomatose intraductal e adenose esclerosante) no tecido mamário adjacente e o tipo de fibroadenoma, complexo ou simples.

\section{Discussão}

Embora a influência etária no aspecto histológico do fibroadenoma seja bem conhecida, sobretudo quanto à tendência de redução do diâmetro, hialinização e calcificação estromal nas mulheres mais idosas, de um modo geral a literatura não tem tratado de maneira detalhada o tema, especialmente no tocante às variações do componente epitelial. Esse último componente foi valorizado por Dupont et al.(9), quando definiram o chamado fibroadenoma complexo e o relacionaram com um discreto aumento do risco relativo para o câncer da mama, comparável ao da hiperplasia ductal típica. O nosso estudo avaliou as características morfológicas dos fibroadenomas nas mulheres em faixas etárias diferentes, na tentativa de estabelecer uma relação temporal evolutiva dessas lesões. Os resultados obtidos são estatisticamente significativos, demonstrando diferenças entre os dois grupos estudados.

Considerando-se o tamanho dos tumores, houve diferenças entre os grupos, pois as mulheres mais velhas apresentaram tumores menores que as mais novas, achado este consistente com a involução dos fibroadenomas com o passar do tempo.

Houve predomínio dos fibroadenomas intracanaliculares sobre os pericanaliculares, diferindo do observado na literatura ${ }^{(14,18)}$. Tal fato é explicável por ser este um material selecionado por grupos etários, com aproximadamente $50 \%$ da casuística formada por mulheres com mais de 40 anos. No entanto, quando separados os grupos 1 e 2, houve predomínio acentuado dos pericanaliculares nas mulheres jovens. Na literatura não se tem enfatizado a associação do padrão pericanalicular à idade mais jovem da paciente. Esse achado do nosso trabalho sugere que, na história natural do fibroadenoma, o estroma vai crescendo ao longo do tempo, até comprimir os ductos, produzindo o padrão fendiforme das lesões intracanaliculares.

Houve diferenças estatísticas da freqüência de características epiteliais indicativas de atividade da lesão, como epitélio normotrófico, adenose comum, hiperplasia ductal e metaplasia apócrina, sendo mais expressivas no grupo 1. Apesar de não ter havido desigualdade estatisticamente comprovada, não se pode deixar de chamar a atenção para o fato de que os três únicos casos de fibroadenomas com setor adenoma-símile estavam no grupo de menor idade.

A elevada freqüência de metaplasia apócrina nos fibroadenomas analisados foi condizente com os dados da literatura ${ }^{(14,21)}$, assim como a relativamente alta freqüência de hiperplasia ductal típica intratumoral (26\%). Neste estudo não encontramos casos de hiperplasia ductal atípica, carcinoma in situ e invasivo, ratificando a raridade de lesões deste tipo dentro do tumor.

Verificou-se diferença estatística entre os grupos etários em relação à celularidade e às alterações estromais. Foram mais freqüentes os tipos normocelular e hipercelular no grupo mais jovem, e o hialinizado no de mais idade. A calcificação predominou no estroma dos fibroadenomas das mulheres mais velhas. A presença de hialinização estromal, associada à diminuição do diâmetro da lesão, com freqüência relacionada ao padrão intracanalicular e ao grupo 2, sugere um continuum de alterações regressivas no qual, após o crescimento máximo do tumor, com oclusão dos ductos, haveria início da atividade involutiva na pós-menopausa. Ocorreriam hialinização crescente do estroma e redução do tamanho da lesão, culminando com sua calcificação. 
Outro dado interessante que obtivemos foi o encontro significativo do estroma mixóide associado a um padrão intracanalicular na totalidade dos casos, mas muito mais significante no grupo mais jovem. Ou seja, nas mulheres jovens o padrão de crescimento tende a ser pericanalicular, exceto quando o estroma é mixóide. Talvez os fibroadenomas mixóides sejam tumores de maior potencial de crescimento. Esse aspecto pode ser sugerido não apenas por produzirem o padrão intracanalicular, mas também pelo fato de termos encontrado lesões significativamente maiores nos tumores mixóides do grupo 1 quando em comparação com os nódulos não-mixóides na totalidade do material. Dentro de uma mesma linha de raciocínio, Tavassoli(18) suspeita de uma provável tendência à recidiva nos tumores mixóides. Outro fato importante nessas lesões é quanto ao relato, na literatura, de que há doenças em que esse tipo de fibroadenoma se associa a mixomas cardíacos e cutâneos como parte do chamado complexo de Carney ${ }^{(6)}$.

A primeira classificação histológica dos tumores da mama da Organização Mundial da Saúde (OMS), publicada em 1968, correlacionava o padrão intracanalicular com um estroma mais freqüentemente do tipo mixóide, que chamou de soft fibroadenoma, em contraste com o hard fibroadenoma, de estroma mais colagenizado, visto, segundo os mesmos autores, como mais prevalente no padrão pericanalicular. No nosso trabalho ficou demonstrado que essa correlação entre estroma mixóide e padrão intracanalicular é mais freqüente no grupo jovem (43\%) do que no mais velho $(17,8 \%)$, predominando o estroma hialinizado, dentro do mesmo padrão, nas mais velhas (68\%) quando em comparação com as mais jovens (18\%). Essa conexão do estroma mixóide com o arranjo intracanalicular feita pelos experts da OMS em 1968 pode ter refletido a maior freqüência de casos diagnosticados como lesões palpáveis (de maior volume) em mulheres jovens numa época em que o rastreio mamográfico ainda não havia sido instituído, pois na verdade o padrão intracanalicular associa-se com mais freqüência ao estroma hialinizado, observando a população em geral. O screening mamográfico preconizado hoje para as mulheres acima de 50 anos está levando a um aumento no diagnóstico destes tumores em fase involutiva, detectados provavelmente pelo achado de calcificações pouco características, indeterminadas, que resultam em cirurgia para diagnóstico.

Outros aspectos pouco freqüentes, mas encontrados nos fibroadenomas desta série, como infiltração e metaplasia adiposas do estroma, cistos e metaplasia dos tipos apócrino e escamoso do epitélio ductal, podem gerar dificuldades diagnósticas em pequenos fragmentos, como os obtidos através das core biopsies. De modo semelhante, os casos em que se verificou área com aspecto phyllodes dentro do fibroadenoma podem induzir ao erro na avaliação. Mas, em correspondência com a literatura ${ }^{(18)}$, que indica maior incidência de tumor phyllodes verdadeiro em mulheres mais velhas, os casos de fibroadenoma com área de aspecto phyllodes encontrados nesta série foram mais freqüentes no grupo mais jovem. Isso deve ser considerado no momento do diagnóstico.

Os achados mostram a grande variedade de alterações intranodulares verificadas nos fibroadenomas ao longo de sua história natural. Algumas dessas modificações já são bem reconhecidas na literatura. Outras foram mais bem esclarecidas no presente estudo, como a associação do padrão de crescimento pericanalicular com as mulheres mais jovens. As classificações mais atuais dos tumores de mama, tanto a da OMS (2003) ${ }^{(20)}$ quanto a do Armed Forces Institute of Pathology (AFIP) (1993)(23), têm desvalorizado a distinção dos fibroadenomas em intra e pericanaliculares por considerá-la sem importância clínica. No entanto, acreditamos que a manutenção dos termos descritivos peri e intracanalicular pode ser útil por refletir características evolutivas da lesão. A definição de fibroadenomas simples e complexos proposta por Dupont et al.(9) foi citada em publicação do Ministério da Saúde ${ }^{(3)}$, embora não tenha sido acatada pela $\mathrm{OMS}^{(20)}$.

\section{Conclusões}

O estudo permite concluir que existem diferenças histopatológicas significantes entre os fibroadenomas de mulheres jovens (grupo 1) e mulheres mais velhas (grupo 2), manifestadas quanto: a) ao diâmetro dos tumores, maior no grupo 1 ; b) ao padrão de crescimento peri e intracanalicular, predominando o primeiro entre as mais jovens e o segundo entre as de mais idade; $c$ ) às alterações que pressupomos indicativas de atividade que prevaleceram no grupo 1(epitélio normotrófico, metaplasia apócrina, adenose comum e hiperplasia ductal típica focal; estroma fibrocelular, tanto normo como hipercelular); d) às alterações de natureza involutiva que predominaram no grupo 2 (epitélio atrófico, normotrófico/atrófico e calcificação intraductal; estroma parcial e totalmente hialinizado e calcificação estromal).

Pode-se concluir, também, que existe importante associação do estroma mixóide com o padrão intracanalicular, principalmente no grupo mais jovem, sugerindo um crescimento mais rápido dessa variante. 


\section{Referências}

I. ABREU-E-LIMA, M. C. C. et al. Sensibilidade e especificidade da core biopsy estereotática no diagnóstico histopatológico das lesões mamárias impalpáveis. Rev Ass Méd Brasil, v. 45, n. 4, p. 290-4, 1999.

2. ABREU-E-LIMA, M. C. C. et al. Aumento da especificidade da mamografia no diagnóstico de lesões impalpáveis:Valor da core biopsy estereotática na exclusão de malignidade. J Bras Patol, v. 36, n. 2, p. I| 8-23, 2000.

3. BRASIL. MINISTÉRIO DA SAÚDE, INSTITUTO NACIONAL DO CÂNCER, COORDENAÇÃO DE PREVENÇÃO E VIGILÂNCIA (CONPREV). Diagnóstico histopatológico e citopatológico das lesões da mama. Rio de Janeiro: CONPREV, 2002. p. 35-7.

4. BLOCK, G. E.; ZLATNIK, P.A. Giant fibroadenomata of the breast in a prepuberal girl. Arch Surg, v. 80, p. I55-9, 1960.

5. CANCER COMMITTEE OF THE COLLEGE OF AMERICAN PATHOLOGISTS. Is "fibrocystic disease" of the breast precancerous? Consensus meeting Oct 3 to 5, 1985, New York. Arch Pathol Lab Med, v. I I0, p. I7I-3, 1986.

6. CARNEY, J.A.;TOORKEY, B. C. Myxoid fibroadenoma and allied conditions (myxomatosis) of the breast:A heritable disorder with special associations including cardiac and cutaneous myxomas. Am J Surg Pathol, v. I 5, n. 8, p. 7| 3-21, I99।.

7. CARTER, B. A. et al. No elevation in long-term breast carcinoma risk for women with fibroadenomas that contain atypical hyperplasia. Cancer, v. I, n. 92( I), p. 30-6, 200 I.

8. DUPONT,W. D.; PAGE, D. L. Risk factors for breast cancer in women with proliferative breast disease. $N$ Engl J Med, v. 312, p. |46-5।, 1985

9. DUPONT,W. D. et al. Long-term risk of breast cancer in women with fibroadenoma. N Engl J Med, v. 331, p. 10-5, 1994.

I0. FEKETE, P. et al. Fibroadenomas with stromal cellularity: a clinical study of 21 patients. Arch Pathol Lab Med, v. I I I, p. 427-32, 1987.

I I. FITZGIBBONS, P.L.; HENSON, D. E.; HUTTER, R.V.P.CANCER COMMITTEE OF THE COLLEGE OF AMERICAN PATHOLOGISTS. Benign breast changes and the risk for subsequent breast cancer: an update of the 1985
Consensus Statement. Arch Pathol Lab Med, v. 122, p. 1053-5, 1998.

12. GESCHICKTER, C. F.; LEWIS, D.; HARTMAN, C. G. Tumors of the breast related to the oestrin hormone. Am J Cancer, v. 21, p. 828-59, 1934.

13. JENSEN, R. A. et al. Invasive breast cancer risk in women with sclerosing adenosis. Cancer, v. 64, p. 1977-83, 1989.

14. KUIJPER, A. et al. Histopathology of fibroadenoma of the breast. Am J Clin Pathol, v. I I 5, p. 736-42, 2001.

15. OBERMAN, H.A. Breast tumors in the adolescent female. Pathol Annu, v. 14; p. 175-201, 1979.

16. PAGE, D. L.;ANDERSON,T.J.; ROGERS, L.W. Epithelial hyperplasia and carcinoma in situ. In: PAGE, D. L.;ANDERSON,T.J. (eds.) Diagnostic histopathology of the breast. Edinburgh, Scotland: Churchill Livingstone, 1987. p. 120-92.

17. ROSEN, P.P.; OBERMAN, H.A.Tumor of the mammary gland. In: Atlas of Tumor Pathology. 3rd series, fascicle 7. Armed Forces Institute of Pathology - Washington, D.C., 1993. p. I0I- 14.

18. TAVASSOLI, F. A. Biphasic tumors. In: TAVASSOLI, F. A. $2^{\text {nd }}$ ed. Pathology of the Breast. Stanford, Connecticut:Appleton and Lange. 1999, p. 57I-98.

19. TAVASSOLI, F. A.; NORRIS, H. J. A comparison of the results of long-term follow-up for atypical intraductal hyperplasia and intraductal hyperplasia of the breast. Cancer, v. 65 , p. 5 | 8-29, 1990.

20. TAVASSOLI, F. K.; DEVILLE, P. World Health Organization Classification of Tumours: Tumours of the Breast and Female Genital Organs. Lion: Ed. IARC, 2003.

21. TOBIAS, P. et al. Revisão histopatológica e análise dos fibroadenomas. Rev Bras Mastol, v. 9. p. 68-74, 1999.

22. UMEKITA, Y.; YOSHIDA, H. Immunohistochemical study of hormone receptor and hormone-regulated protein expression in phyllodes tumor: comparison with fibroadenoma. Virchows Arch, v. 433, p. 31 I-4, 1998.

23. WEIND, K. L. et al. Invasive carcinomas and fibroadenomas of the breast: comparison of microvessel distributions. Implications for imaging modalities. Radiology, v. 208, p. 477-83, 1988. 